ARTICLE

httpst//doi.org/10.1038/s414.67-020-15239-3

\title{
Plant Raf-like kinases regulate the mRNA population upstream of ABA-unresponsive SnRK2 kinases under drought stress
}

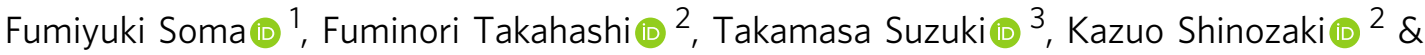 \\ Kazuko Yamaguchi-Shinozaki id ${ }^{1 凶}$
}

SNF1-related protein kinases 2 (SnRK2s) are key regulators governing the plant adaptive responses to osmotic stresses, such as drought and high salinity. Subclass III SnRK2s function as central regulators of abscisic acid (ABA) signalling and orchestrate ABA-regulated adaptive responses to osmotic stresses. Seed plants have acquired other types of osmotic stress-activated but ABA-unresponsive subclass I SnRK2s that regulate mRNA decay and promote plant growth under osmotic stresses. In contrast to subclass III SnRK2s, the regulatory mechanisms underlying the rapid activation of subclass I SnRK2s in response to osmotic stress remain elusive. Here, we report that three B4 Raf-like MAP kinase kinase kinases (MAPKKKs) phosphorylate and activate subclass I SnRK2s under osmotic stress. Transcriptome analyses reveal that genes downstream of these MAPKKKs largely overlap with subclass I SnRK2-regulated genes under osmotic stress, which indicates that these MAPKKKs are upstream factors of subclass I SnRK2 and are directly activated by osmotic stress.

\footnotetext{
${ }^{1}$ Laboratory of Plant Molecular Physiology, Graduate School of Agricultural and Life Sciences, The University of Tokyo, Bunkyo-ku, Tokyo 113-8657, Japan. ${ }^{2}$ Gene Discovery Research Group, RIKEN Center for Sustainable Resource Science, Tsukuba, Ibaraki 305-0074, Japan. ${ }^{3}$ College of Bioscience and

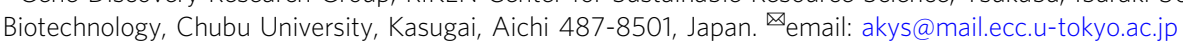


biotic stresses, particularly osmotic stresses such as drought and high salinity, are adverse environmental factors for plant growth and crop productivity. The plant hormone abscisic acid (ABA) plays important roles in the adaptive responses to these stresses. The proteins belonging to the SNF1-related protein kinase 2 (SnRK2) family include key regulators of osmotic stress responses, and are classified into three subclasses $^{1-3}$. Among these subclasses, subclass III SnRK2 proteins are strongly activated by $\mathrm{ABA}$ and act as essential positive regulators of $\mathrm{ABA}$ signalling downstream of $\mathrm{ABA}$ receptors, such as pyrabactin-resistance1/PYR1-like/regulatory components of $\mathrm{ABA}$ receptors (PYR/PYL/RCARs), under osmotic stress conditions ${ }^{4-10}$. The activated subclass III SnRK2s modulate various $\mathrm{ABA}$ responses, including the induction of stress-responsive genes, through activation of the bZIP-type transcription factors ABA-responsive element (ABRE)-binding proteins/ABRE-binding factors (AREB/ABFs) ${ }^{11-15}$. In plants, subclass III SnRK2s are activated in response to $\mathrm{ABA}$ and osmotic stress in not only an ABA-dependent but also an ABA-independent manner ${ }^{3,16}$. Seed plants have acquired other types of SnRK2s in addition to subclass III SnRK2s, specifically, subclass I SnRK2s, such as SRK2A/ SnRK2.4, SRK2B/SnRK2.10, SRK2G/SnRK2.1 and SRK2H/ SnRK2.5, which are rapidly activated by osmotic stress prior to ABA accumulation and, unlike subclass III SnRK2s, are not activated by $\mathrm{ABA}^{3,16}$.

Phenotypic analyses of Arabidopsis mutants defective in some subclass I SnRK2s have revealed that ABA-unresponsive subclass I SnRK2s are involved in plant growth and survival under osmotic stress conditions ${ }^{17-19}$, but the functional analysis of these SnRK2s has been delayed compared with that of subclass III SnRK2s. To elucidate the physiological function of subclass I SnRK2-mediated phosphorylation signalling, subclass I SnRK2interacting proteins were recently analysed by co-IP coupled with LC-MS/MS analysis ${ }^{20}$. Several components of the mRNAdecapping complex, such as VARICOSE (VCS) and DECAPPING 2 (DCP2) ${ }^{21,22}$, have been identified as candidate interacting proteins. Among these components, VCS, which is a mRNAdecapping activator, physically interacts with four functional subclass I SnRK2s but not with subclass III SnRK2s, and is phosphorylated by all four functional subclass I SnRK2s in processing bodies (P-bodies) under osmotic stress conditions. A mutant plant with no functional subclass I SnRK2s and VARICOSE-knockdown plants exhibit growth retardation under osmotic stress conditions. The expression of many stressresponsive genes is similarly dysregulated in these plants, and the mRNA decay of these transcripts is decreased in these plants under osmotic stress conditions. Subclass I-type SnRK2s have been identified in seed plants, but not in lycophytes or mosses. The "ABA-activated subclass III SnRK2s-AREB/ABF" signalling module is known to function as the transcriptional activation system in ABA-responsive gene expression in from mosses to seed plants ${ }^{23}$. To enhance their adaptability under constitutively changing stress conditions ${ }^{20}$, seed plants are thought to have acquired a post-transcriptional regulation system mediated by the "ABA-unresponsive subclass I SnRK2s-VARICOSE" signalling module, which promotes marked changes in the mRNA populations under osmotic stress conditions, in addition to the transcriptional activation system during the process of evolution.

Although the mechanisms underlying the activation of ABAactivated subclass III SnRK2s have been clarified in detail, the mechanisms responsible for the rapid activation of ABAunresponsive subclass I SnRK2s in response to osmotic stress remain largely unknown, despite their pivotal roles in adaptive responses under osmotic stress conditions. Here, we attempt to identify novel interactors of subclass I SnRK2s through co-IP coupled with LC-MS/MS analysis. Three B4 Raf-like MAP kinase kinase kinases (B4 Raf-like MAPKKKs) are identified as upstream factors of the ABA-unresponsive subclass I SnRK2-VCS signalling pathway that are activated by osmotic stress.

\section{Results}

Identification of subclass I SnRK2-interacting proteins. To increase the current understanding of ABA-independent osmotic stress signalling, we first attempted to identify ABA-unresponsive subclass I SnRK2-interacting proteins in Arabidopsis. Among the four members of the functional subclass I SnRK2s, we selected SRK2A and SRK2G due to their strong expression in root and shoot tissues ${ }^{20}$. Green fluorescent protein (GFP)-tagged SRK2A (SRK2A-GFP) and SRK2G (SRK2G-GFP) were immunoprecipitated from SRK2A-GFP- and SRK2G-GFP-expressing plants treated with $800 \mathrm{mM}$ mannitol for $10 \mathrm{~min}$, respectively. The immunoprecipitates were subsequently analysed using liquid chromatography-tandem mass spectrometry (LC-MS/MS). Each of these LC-MS/MS analyses, using the SRK2A-GFP- and SRK2G-GFP-expressing plants, was performed twice as biological replicates. We excluded the proteins that were detected in GFPexpressing plants from the analytical results. The LC-MS/MS analyses ultimately identified 1308 and 774 proteins (with confidence $>95 \%$ ) as candidate SRK2A-GFP- and SRK2G-GFPinteracting proteins, respectively (Fig. 1a). A previous study revealed that the phosphorylation/dephosphorylation of subclass I SnRK2s is involved in their activation ${ }^{24}$. We thus focused on 21 protein kinases and five phosphatases among the 731 candidate proteins detected in both the immunoprecipitate from the SRK2A-GFP-expressing plants and that from the SRK2G-GFPexpressing plants for our investigation of upstream factors of the subclass I SnRK2s (Fig. 1a; Supplementary Data 1). Interestingly, we found that three B4 Raf-like MAPKKKs were included in these 26 candidate proteins (Supplementary Data 1). Previous studies showed that these three Raf-like kinases were phosphorylated during the early stages of the osmotic stress response of plants ${ }^{25}$. We then focused on these three Raf-like kinases RAF18, RAF20 and RAF24.

To further investigate the physical interactions between subclass I SnRK2s and the three Raf-like kinases, we conducted a co-immunoprecipitation (co-IP) assay using untreated or mannitol-treated plants expressing both RAF18-GFP and SRK2A-mCherry, SRK2G-mCherry SRK2D/SnRK2.2-mCherry. RAF18 was used for the assay as a representative of the three Raflike kinases. We observed that the SRK2A-mCherry and SRK2GmCherry proteins were coimmunoprecipitated with the RAF18GFP protein, but not with the SRK2D-mCherry protein in extracts from both untreated and mannitol-treated plants (Fig. 1b). We subsequently performed a split-luciferase complementation (Split-LUC) assay using Nicotiana benthamiana leaves. Luciferase signals were detected when SRK2A-cLUC and RAF18nLUC, RAF20-nLUC or RAF24-nLUC were transiently expressed, but not when both nLUC and SRK2A-cLUC were expressed (Fig. 1c). We also performed a split-LUC experiment using SRK2G-cLUC and detected luciferase signals when SRK2GcLUC and RAF18-nLUC, RAF20-nLUC or RAF24-nLUC were expressed (Supplementary Fig. 1). These results suggest that RAF18, RAF20 and RAF24 are novel potential candidate interacting proteins with subclass I SnRK2s.

Because subclass I SnRK2s localise to P-bodies under osmotic stress conditions ${ }^{20}$, the three Raf-like kinases might physically interact with subclass I SnRK2s in P-bodies under osmotic stress conditions. We analysed the subcellular localisation of RAF18 and found that RAF18-GFP mainly localised to the cytoplasm after water treatment (control), whereas a portion of RAF18-GFP accumulated in punctate structures in response to mannitol and 
a

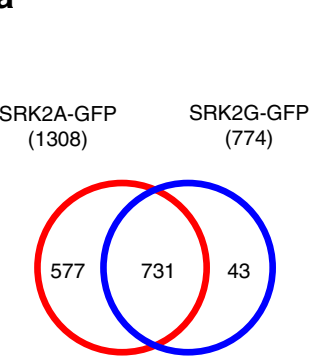

b
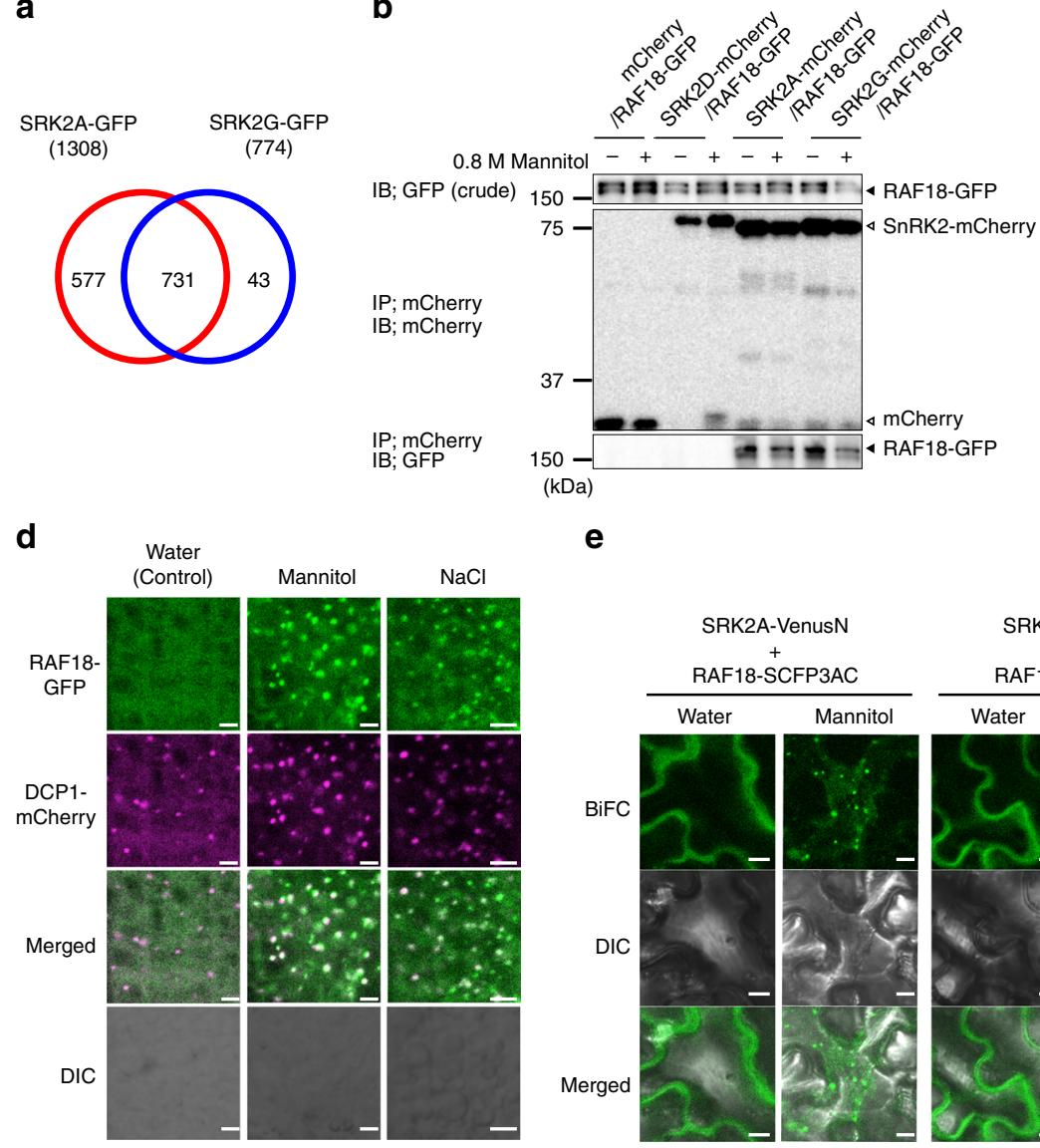

e

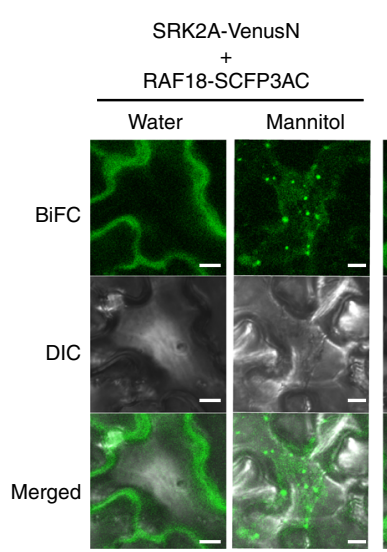

C

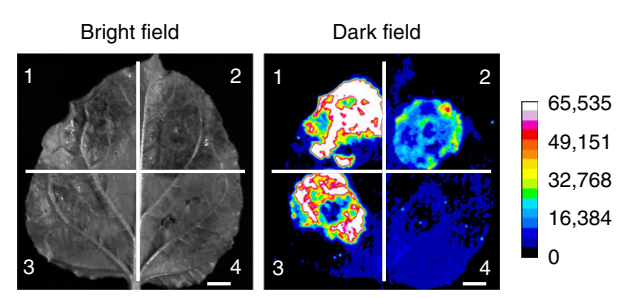

1. RAF18-nLUC + SRK2A-cLUC

2. RAF20-nLUC + SRK2A-CLUC

3. RAF24-nLUC + SRK2A-cLUC

4. $n L U C+$ SRK2A-CLUC

Fig. 1 The three Raf-like kinases localise and physically interact with subclass I SnRK2s in P-bodies under osmotic stress conditions. a Venn diagrams showing the numbers of candidate SRK2A-GFP- or SRK2G-GFP-interacting proteins detected in two independent samples under osmotic stress conditions. Each of the analyses was performed twice as biological replicates. $\mathbf{b}$ The physical interactions of the three Raf-like kinases with subclass I SnRK2s were validated through a co-immunoprecipitation (co-IP) assay. Co-IP was performed using total proteins extracted from transgenic Arabidopsis plants coexpressing RAF18-GFP and mCherry, SRK2A-mCherry, SRK2G-mCherry or SRK2D-mCherry that were treated or not treated with 0.8 M mannitol for 10 min using an anti-mCherry antibody. Immunoblotting was performed with an anti-GFP or anti-mCherry antibody. c Split-luciferase assay of the interaction between the three Raf-like kinases and SRK2A in infiltrated Nicotiana benthamiana leaves; the bright-field (left) and dark-field (right) results are shown. Scale bars, $1 \mathrm{~cm}$. d Confocal images of GFP fluorescence in root cells of transgenic Arabidopsis expressing both RAF18-GFP and DCP1-mCherry and treated with water, $500 \mathrm{mM}$ mannitol or $250 \mathrm{mM} \mathrm{NaCl}$ for $30 \mathrm{~min}$. Scale bars, $5 \mu \mathrm{m}$. e BiFC analyses of the physical interactions between SnRK2s and RAF18 in $\mathrm{N}$. benthamiana leaves expressing both SRK2A- or SRK2G-VenusN and RAF18-SCFP3AC and treated with water or 500 mM mannitol for $5 \mathrm{~h}$. Scale bars, $10 \mu \mathrm{m}$. f Confocal images of fluorescent proteins in $N$. benthamiana leaves expressing SRK2A-VenusN, RAF18-SCFP3AC and DCP1-mCherry. Scale bars, 10 $\mu$ m.

$\mathrm{NaCl}$ treatments (Fig. 1d). Furthermore, punctate RAF18-GFP signals largely overlapped with the signals from the P-body marker DCP1-mCherry (Fig. 1d). These observations suggested that RAF18 localised to P-bodies under osmotic stress conditions. We then validated the physical interaction between subclass I SnRK2s and the three Raf-like kinases at a subcellular level. Bimolecular fluorescence complementation (BiFC) assays showed that the Raf-like kinases interacted with SRK2A and SRK2G in the cytoplasm after water treatment, whereas no detectable interaction between these proteins and MPK6 was found (Fig. 1e; Supplementary Fig. 2). We subsequently performed a BiFC assay under osmotic stress conditions, and detected interactions between RAF18 and SRK2A or SRK2G in punctate structures (Fig. 1e). Furthermore, punctate signals indicating an interaction between RAF18 and SRK2A largely overlapped with DCP1mCherry signals under osmotic stress conditions (Fig. 1f), which indicated that RAF18 physically interacts with subclass I SnRK2s in P-bodies under osmotic stress conditions.

Previous studies have revealed that subclass I SnRK2s are highly conserved in seed plants, but not in lycophytes or mosses ${ }^{20}$. Therefore, we analysed the phylogenetic relationship among the Raf-like kinases in various plant species. A molecular phylogenetic analysis revealed that RAF18 (AT1G16270), RAF20 (AT1G79570) and RAF24 (AT2G35050) belong to the group of B4 MAPKKKs ${ }^{26}$ (Supplementary Fig. 3). The group of B4 MAPKKKs was widely conserved from mosses to seed plants, whereas RAF18/20/24 have been identified in seed plants, including Oryza sativa and Solanum lycopersicum, but not in lycophytes and mosses (Supplementary Fig. 3). Based on these results, the thee Raf-like kinases and subclass I SnRK2s might have been specifically acquired by seed plants.

We then evaluated the tissue-specific expression profiles of the three $R A F$ genes through the generation of transgenic Arabidopsis plants carrying the promoter of RAF18, RAF20 or RAF24 fused to the GUS gene. GUS activity was widely observed in both the aerial parts and roots of the RAF18pro:GUS, RAF20pro:GUS and RAF24pro:GUS plants, which suggested that the three $R A F$ genes are broadly expressed in vegetative tissues (Supplementary Fig. 4a). The expression of $R A F$ genes was further validated by quantitative reverse transcription-polymerase chain reaction (quantitative RT-PCR). In addition to the results from the GUS analyses, the three $R A F$ genes were widely expressed in both the 
aerial parts and roots of the wild-type plants (Supplementary Fig. 4b).

The three Raf-like kinases activate subclass I SnRK2s. Importantly, subclass I SnRK2s are rapidly phosphorylated and activated in response to osmotic stress, but the responsible kinases remain unknown. We thus examined whether the three Raf-like kinases could phosphorylate subclass I SnRK2s in vitro. The recombinant glutathione $S$-transferase (GST)-tagged RAF18 kinase domain (RAF18KD-GST), RAF20 kinase domain (RAF20KD-GST) and RAF24 kinase domain (RAF24KD-GST) enhanced the phosphorylation status of recombinant maltosebinding protein (MBP)-tagged SRK2A (SRK2A-MBP), SRK2B (SRK2B-MBP), SRK2G (SRK2G-MBP) and SRK2H (SRK2HMBP) in vitro (Fig. 2a; Supplementary Fig. 5a). However, we could not evaluate whether the three Raf-like kinases phosphorylate subclass I SnRK2s or enhance their autophosphorylation activities. Therefore, we generated kinase-inactive forms of subclass I SnRK2s because their autophosphorylation activities made it difficult to assess the possibility of transphosphorylation events between these protein kinases. Lys-33 of SRK2A corresponds to a highly conserved residue that is required for the activity of SnRK2 $\mathrm{s}^{27}$. Although the mutation of Lys-33 in subclass I SnRK2s to Asn, which produced SRK2A (SRK2AKN), SRK2B (SRK2BKN), SRK2G (SRK2GKN) and SRK2H (SRK2HKN), abolished their autophosphorylation activities (Fig. 2b; Supplementary Fig. 5b), all the SRK2AKN-MBP, SRK2BKN-MBP, SRK2GKN-MBP and SRK2HKN-MBP proteins were phosphorylated by RAF18KD-GST, RAF20KD-GST and RAF24KDGST (Fig. 2b; Supplementary Fig. 5b). These observations indicated that the three Raf-like kinases can phosphorylate subclass I SnRK2s in vitro. Because VCS was previously shown to be phosphorylated by subclass I SnRK2s $s^{20}$, in-gel kinase assays of autophosphorylated and RAF20-phosphorylated SRK2A-MBP or SRK2G-MBP were performed using a maltose-binding protein (MBP)-fused VCS fragment (VCSm-MBP) as the substrate to examine the effects of the phosphorylation of subclass I SnRK2s by the Raf-like kinases on these kinase activities. RAF20phosphorylated SRK2A-MBP and SRK2G-MBP exhibited stronger kinase activities than autophosphorylated SRK2A-MBP and SRK2G-MBP, respectively (Fig. 2c). These data indicate that subclass I SnRK2s are activated through their phosphorylation catalysed by the three Raf-like kinases in vitro.

To determine the sites in subclass I SnRK2s that are phosphorylated by the three RAFs, RAF20-phosphorylated and autophosphorylated SRK2G-MBP as shown in Fig. 2c were analysed by mass spectrometry. We focused on the phosphorylation sites detected in RAF20-phosphorylated SRK2G-MBP, but not autophosphorylated SRK2G-MBP. Nine phosphorylation sites in SRK2G specific to RAF20-phosphorylated SRK2G-MBP were identified (Supplementary Data 2). Among these phosphorylation sites, Ser-154 in SRK2G appears to be an important target residue because it was the first serine in the SxxxS/T motif, which has been shown to be a typical phosphorylation site targeted by various MAPKKKs ${ }^{28}$. Moreover, this Ser-154 residue was conserved among all four subclass I SnRK2s (Supplementary Fig. 6a), and the residue in SRK2B is reportedly phosphorylated in response to osmotic stress ${ }^{29}$. We then performed an analysis of the mutation of Ser-154 in SRK2GKN-MBP to Ala, which resulted in abolishment of the autophosphorylation activity (Fig. 2b; SRK2GKN). The phosphorylation level of SRK2GKN_S154A-MBP by RAF20KD-GST was clearly decreased compared with that of SRK2GKN-MBP (Supplementary Fig. 6b), which suggests that the Ser-154 of SRK2G is one of the direct phosphorylation targets of the three Raf-like kinases.
To elucidate whether subclass I SnRK2s are phosphorylated and activated by the three Raf-like kinases in vivo, we generated a triple mutant of the three $R A F$ genes, raf18/20/24, by crossing three T-DNA insertion mutants of each gene to one another. A RT-PCR analysis confirmed that the raf18/20/24 mutant plants exhibited decreased expression of RAF20 and did not express the other RAF18 and RAF24 (Supplementary Fig. 7a,b). The growth phenotypes of the raf18/20/24 mutant plants grown on agar plates were similar to those of the wild-type plants (Supplementary Fig. 7c, d, e). We then performed an in-gel kinase assay to analyse the kinase activities of subclass I SnRK2s in the raf single or multiple mutants. The kinase activity of native subclass I SnRK2s in response to dehydration stress was significantly decreased in the raf18/20/24 mutant plants, but not in the single- or doublemutant plants (Fig. 2d; Supplementary Fig. 8a). Our in-gel kinase assay also showed that the activation of native subclass I SnRK2s in response to high salinity or mannitol treatment was largely impaired in the raf18/20/24 mutant plants (Supplementary Fig. 8b). These in-gel kinase assays also found some unknown VCS-phosphorylating kinase activity other than that of SnRK2s (Fig. 2d; Supplementary Fig. 8; asterisk), which was likely due to at least one mitogen-activated protein (MAP) kinase based on the detected molecular weight. The impaired activation of subclass I SnRK2s in the raf18/20/24 mutant plants was recovered by the expression of RAF18, RAF20 or RAF24 (Supplementary Fig. 9). Taken together, these results suggest that the three RAFs redundantly and positively regulate the activity of subclass I SnRK2s under osmotic stress conditions in vivo.

We subsequently analysed the phosphorylation status of SRK2B-GFP, which is a subclass I SnRK2, in wild-type or raf18/20/24 mutant plants because dehydration stress induced clearer electrophoretic mobility shifts of SRK2B-GFP mediated by phosphorylation compared with those obtained with any other subclass I SnRK2s ${ }^{20}$. Clear electrophoretic mobility shifts and activation of SRK2B-GFP were observed in the wild-type plants in response to dehydration stress, but not ABA treatment (Fig. 2e). The shifted bands were confirmed to be phosphorylated forms through treatment with a $\lambda$ protein phosphatase ( $\lambda$ PP) (Fig. 2e). In contrast, the activation of SRK2D-GFP, which is an ABAresponsive subclass III SnRK2, was observed in response to both dehydration stress and $\mathrm{ABA}$ treatment in the wild-type plants (Fig. 2e). The clear electrophoretic mobility shifts and activation of SRK2B-GFP in response to dehydration were largely impaired in the raf18/20/24 mutant plants (Fig. 2f). In contrast, the activation of SRK2D-GFP in response to both dehydration stress and ABA treatment observed in the raf18/20/24 mutant plants was similar to that found in the wild-type plants (Fig. 2f). These results suggest that the three Raf-like kinases activate subclass I SnRK2s via their phosphorylation in vivo.

We then performed an in-gel kinase assay to analyse the native kinase activities of subclass III SnRK2s in raf18/20/24 mutant plants using histone, which can be phosphorylated by both subclass I and III SnRK2s, as the substrate ${ }^{20}$. Several phosphorylated bands were observed in response to ABA treatment in the wild-type plants. However, some of the phosphorylated bands were impaired in the srk2dei mutant plants that lack all subclass III SnRK2s (SnRK2D/SnRK2.2, SnRK2E/SnRK2.6 and SnRK2I/ SnRK2.3; Fig. 2g), but the phosphorylated bands obtained by subclass III SnRK2s in response to ABA treatment were not affected in the raf18/20/24 mutant plants (Fig. $2 \mathrm{~g}$ ). We checked the activity of subclass III SnRK2s in response to dehydration in the raf18/20/24 mutant plants. Several phosphorylated bands were observed in response to dehydration stress in the wild-type plants, but some of these bands were impaired in the srk2dei mutant plants (Fig. 2h), and the bands phosphorylated by subclass III SnRK2s were not changed in the raf18/20/24 mutant 
a

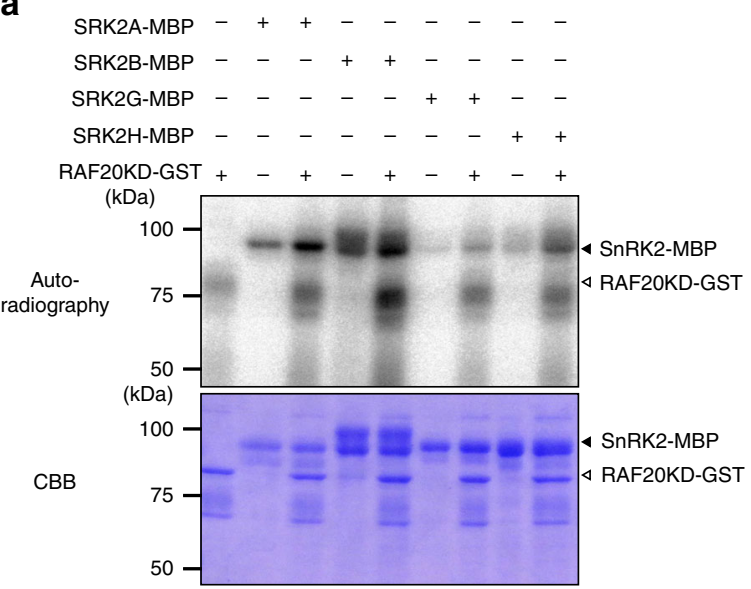

C

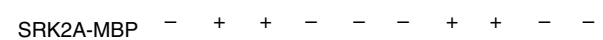

SRK2G-MBP $-\quad-++-\quad++$

RAF20KD-GST +-+-++-++

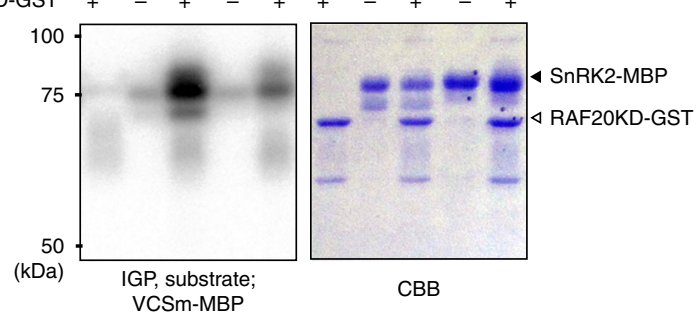

e

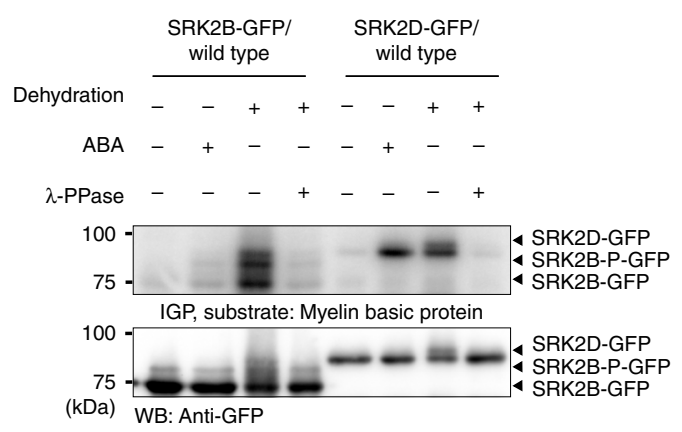

9

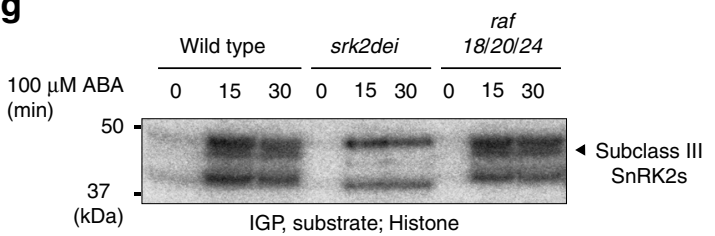

b

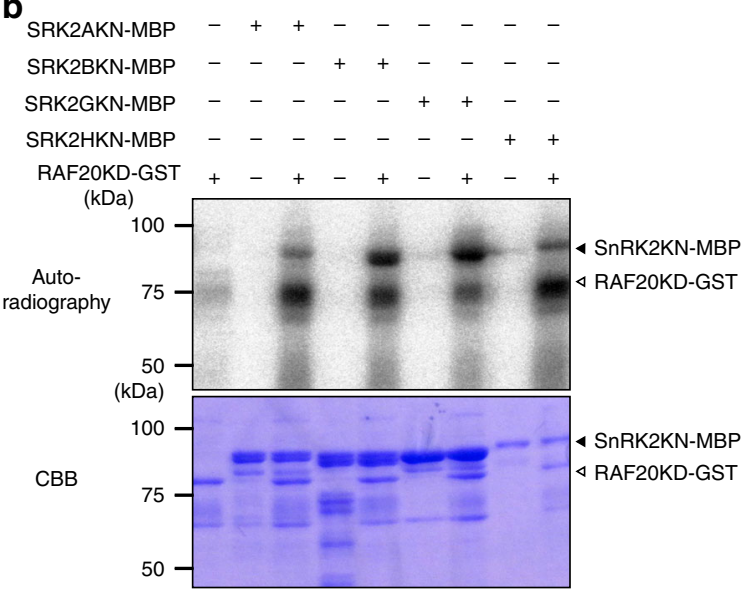

d

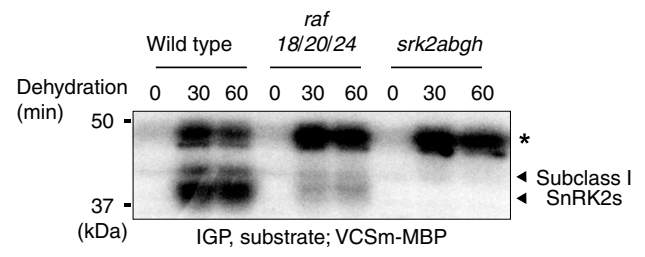

$\mathbf{f}$

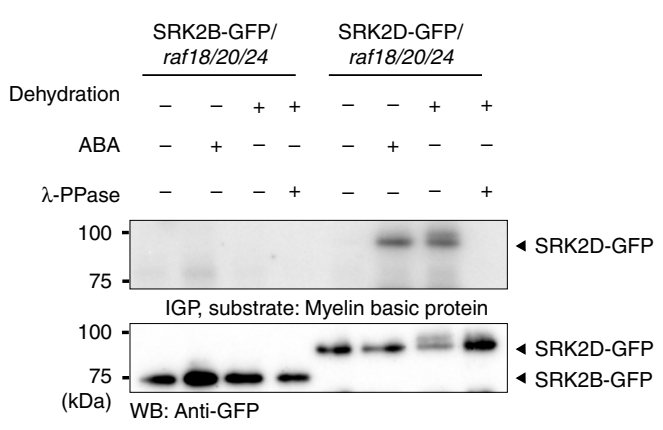

h

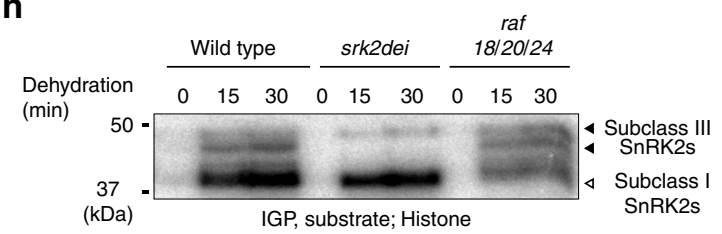

Fig. 2 The three Raf-like kinases phosphorylate and activate subclass I SnRK2s under osmotic stress conditions. a Phosphorylation of SRK2A-MBP, SRK2B-MBP, SRK2G-MBP or SRK2H-MBP by RAF2OKD-GST in vitro; 500 ng of the recombinant proteins was reacted with g-[32P]ATP, electrophoresed on an SDS-polyacrylamide gel and then subjected to Coomassie Brilliant Blue (CBB) staining and autoradiography (32P). b Phosphorylation of SRK2AKNMBP, SRK2BKN-MBP, SRK2GKN-MBP or SRK2HKN-MBP by RAF20-GST in vitro. The phosphorylation assays were performed as described in a. $\mathbf{c}$ The phosphorylation of VCSm by SRK2A-MBP or SRK2G-MBP that was in vitro phosphorylated by RAF2OKD-GST. In-gel kinase assays (IGP) of recombinant SRK2A-MBP ( $500 \mathrm{ng}$ ) or SRK2G-MBP (500 ng) phosphorylated by RAF20KD-GST (500 ng). The CBB staining and autoradiography results are shown. VCSm-MBP was used as the substrate. d IGP assay of VCSm phosphorylation using crude extracts from wild-type, raf18/20/24 and srk2abgh plants in response to drought stress. VCSm-MBP was used as the substrate. An asterisk indicates unknown kinase(s) that can phosphorylate VCSm-MBP. e, $\mathbf{f}$ Electrophoretic mobility and phosphorylation activities of SRK2B-GFP or SRK2D-GFP in wild-type (e) and raf18/20/24 plants (f) in response to dehydration stress or ABA treatment. The proteins were purified using an anti-GFP antibody from transgenic Arabidopsis expressing SRK2B-GFP or SRK2D-GFP and subjected to dehydration stress or ABA treatment for 30 min were analysed by immunoblotting (IB) with an anti-GFP or in-gel kinase assay (IGP) using myelin basic protein as the substrate. The electrophoretic mobility of SRK2B-GFP or SRK2D-GFP in response to dehydration after treatment with protein phosphatase ( $\lambda$-PPase) is also shown. $\mathbf{g}$, $\mathbf{h}$ IGP assay of histone using crude extracts from wild-type, srk2dei and raf18/20/24 plants in response to $A B A$ treatment $(\mathbf{g})$ or dehydration stress $(\mathbf{h})$. 
plants (Fig. 2h). In contrast, the bands phosphorylated by kinases other than subclass III SnRK2s, which appear to be subclass I SnRK2s, were decreased in the raf18/20/24 mutant plants. These observations indicate that the three Raf-like kinases regulate subclass I SnRK2s under osmotic stress conditions but are not involved in the regulation of subclass III SnRK2s and responses to ABA treatment.

RAF18/20/24 regulate plant growth under osmotic stress. To gain insight into the functional relationships between the three Raf-like kinases and subclass I SnRK2s in plants, we examined the growth of the raf mutants under osmotic stress-inducing conditions. Our previous studies showed that srk2abgh plants lacking all functional subclass I SnRK2 genes showed growth retardation under osmotic stress conditions ${ }^{20}$. When grown in soil with a limited water supply, the raf single- and double-mutant plants showed a similar growth phenotype as that of the wild-type plants (Supplementary Fig. 10). In contrast, under drought stress conditions, the raf18/20/24 triple-mutant plants showed growth retardation with smaller leaves compared with the wild-type plants, similar to the effects observed in the srk2abgh plants (Fig. 3a, b; Supplementary Fig. 10). The enhanced growth retardation detected in the raf18/20/24 plants was recovered by complementation of the RAF18, RAF20 or RAF24 genes (Fig. 3a, b; Supplementary Fig. 11). Because the RAF18/20/24-subclass I SnRK2 signalling module regulates plant growth under osmotic stress conditions, the growth of raf18/20/24 plants in soil might be affected under low-humidity conditions. When grown in soil under $80 \%$ humidity (relative humidity; $\mathrm{RH}, 22^{\circ} \mathrm{C}$ ), both the raf18/20/24 and srk2abgh plants grew similarly to the wild-type plants (Supplementary Fig. 12). In contrast, when grown under $50 \%$ humidity, both mutant plants showed retarded growth compared with the wild-type plants (Supplementary Fig. 12). We subsequently examined the growth of the raf18/20/24 plants on agar plates under high salinity or osmotic stress conditions. In the presence of $100 \mathrm{mM} \mathrm{NaCl}$ or $100 \mathrm{mM}$ mannitol, the raf singleand double-mutant plants showed similar growth (Supplementary Fig. 13), whereas the srk2abgh and raf18/20/24 plants showed retarded growth relative to the wild-type plants, as demonstrated by smaller aerial parts and reduced primary root growth (Fig. 3c-e; Supplementary Fig. 13). The enhanced growth retardation detected in the raf18/20/24 plants was recovered through expression of the RAF18, RAF20 or RAF24 gene (Fig. 3c-e; Supplementary Fig. 14). These observations suggest that the RAF18/20/24-subclass I SnRK2 signalling module plays important roles in plant growth under osmotic stress conditions. In most of the plant growth experiments, the raf18/20/24 plants showed slightly retarded growth compared with the srk2abgh plants, which implies the existence of other minor targets of the three Raf-like kinases.

RAF18/20/24 regulates osmotic stress-responsive genes. Previous studies have revealed that subclass I SnRK2s facilitate marked changes in the population of mRNAs that regulate mRNA decay via phosphorylation of a mRNA-decapping activator, VCS, under osmotic stress conditions ${ }^{20}$. Because the subclass I SnRK2s-VCS module promotes changes in the mRNA population, the three Raf-like kinases might also play roles in regulating the mRNA population under osmotic stress. To examine this hypothesis, we performed transcriptomic analyses of the wild-type, raf18/20/24 and srk2abgh plants following dehydration stress treatment through RNA-sequencing analysis. After dehydration treatment for $5 \mathrm{~h}, 400$ and 989 genes significantly increased and decreased in expression levels, respectively, in the raf18/20/24 plants compared with those in the wild-type plants (fold change $>2$ or fold change $<0.5, P<0.05$ ) (Fig. 4a, b; Supplementary Data 3). Importantly, many of the up- and downregulated genes in the raf18/20/24 under dehydration stress were similarly regulated in the srk2abgh plants (Fig. $4 \mathrm{a}, \mathrm{b}$ ). In addition, the expression levels of the up- and downregulated genes in the raf18/20/24 plants were significantly correlated with their levels in the srk2abgh plants, respectively (Fig. 4c). Furthermore, the majority of up- and downregulated genes in the raf18/20/24 plants were repressed and induced by dehydration, respectively, in the wild-type plants (Fig. 4d). The increased or decreased expression of genes in the raf18/20/24 plants was further validated by quantitative RT-PCR. mRNA transcripts, such as CYP75B1, MLP423, CYSTM8 and At1g13609, which were repressed in the wild-type plants by dehydration stress, accumulate to higher levels in both the raf18/20/24 and srk2abgh plants under dehydration stress conditions compared with their levels in the wildtype plants (Fig. 4e). In contrast, various mRNA transcripts, such as ERF53, ERF54, At5g25600 and SIS, which were induced by dehydration in the wild-type plants, accumulated to lower levels in both raf18/20/24 and srk2abgh plants under dehydration stress compared with their levels in the wild-type plants (Fig. 4f). These results suggest that the three Raf-like kinases regulate the mRNA population under osmotic stress by activating subclass I SnRK2 activity.

\section{Discussion}

Although subclass I SnRK2s are activated in response to osmotic stress and function in the regulation of mRNA decay, the associated activation mechanism has not been elucidated ${ }^{20}$. Unlike subclass III SnRK2s, these kinases are not activated by ABA and are thought to be directly and promptly activated by osmotic stress prior to ABA accumulation. We thus attempted to elucidate the subclass I SnRK2-mediated signalling system in comparison with the subclass III SnRK2 signalling system. Since subclass I SnRK2s are rapidly activated in response to osmotic stress, we performed LC-MS/MS analyses to identify subclass I SnRK2interacting proteins in plants that were treated with $800 \mathrm{mM}$ mannitol for a short time $(10 \mathrm{~min})$ (Fig. 1a). Considering the possible existence of an activation mechanism that involves the phosphorylation cascade, we focused on 21 protein kinases and five phosphatases which were among the identified candidate interacting proteins (Supplementary Data 1). The data from the previous phosphoproteomic analyses ${ }^{25}$ revealed that three B4 Raf-like MAPKKKs, denoted RAF18, RAF20 and RAF24, among the 21 candidate protein kinases were phosphorylated within 10 min in response to osmotic stress. Furthermore, we performed a subcellular localisation analysis, in vitro phosphorylation assays and investigations of plants with multiple mutations in RAF18, RAF20 and RAF24 and found that these three Raf-like kinases were protein kinases that phosphorylate and activate subclass I SnRK2s in Arabidopsis plants under osmotic stress conditions (Figs. 1 and 2). In addition, we found that the three Raf-like kinases are not activated by ABA and are not involved in the activation of subclass III SnRK2s, which suggests that the RAF18/ 20/24-subclass I SnRK2-VCS signalling pathway acts independently from the subclass III SnRK2 signalling pathway. Thus, this study highlights the existence of three Raf-like kinases that activate downstream subclass I SnRK2s in response to osmotic stress to form the ABA-independent RAF18/20/24-subclass I SnRK2VCS signalling cascade (Fig. $4 \mathrm{~g}$ ).

The three Raf-like kinases belong to the family of B4 Raf-like MAPKKKs. Eighty MAPKKKs have been identified in the Arabidopsis genome, and these have been classified into three groups denoted A to C. Among the 80 MAPKKKs, 48 MAPKKKs classified into groups $\mathrm{B}$ and $\mathrm{C}$ are Raf-like kinases. In addition, the 
a

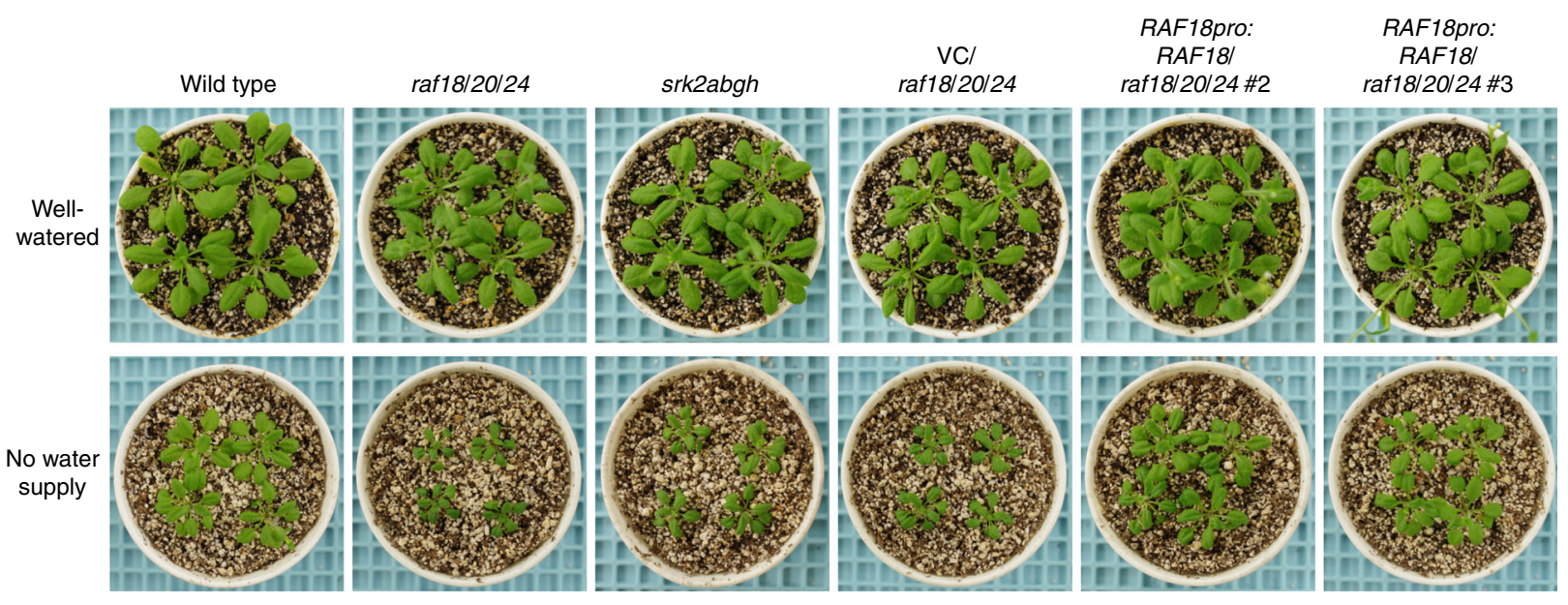

b

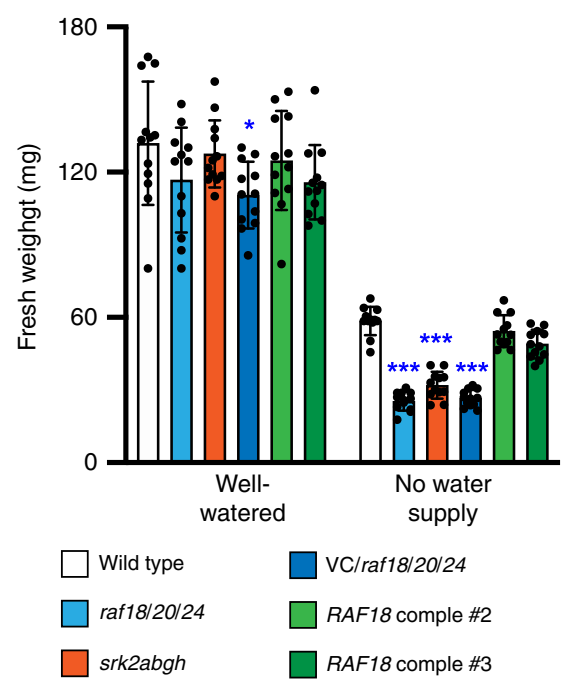

d

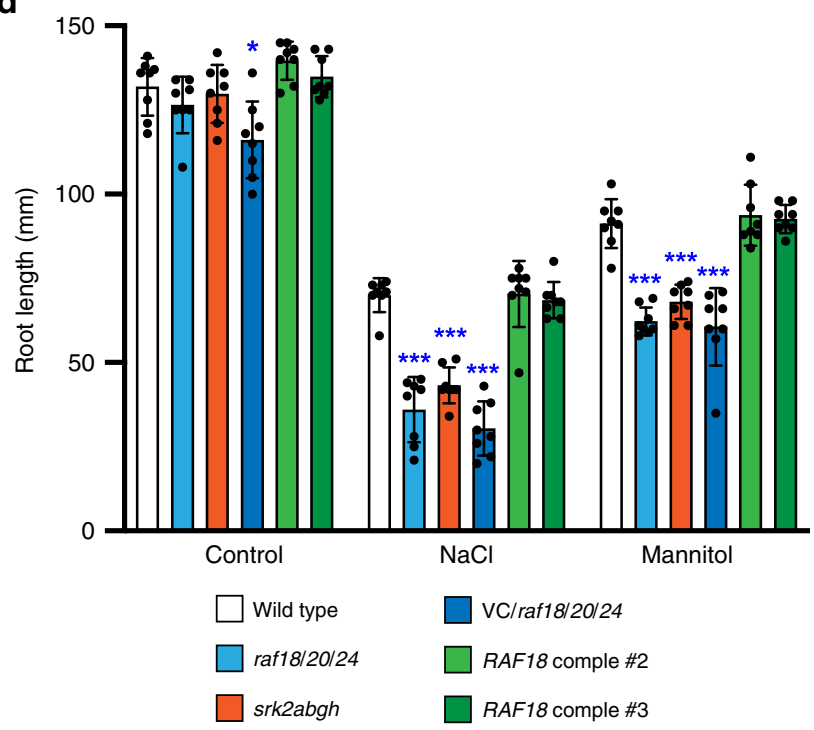

Raf-like MAPKKKs classified into group B are divided into four subgroups denoted B1 to B4 ${ }^{26,30}$ (Supplementary Fig. 3). The B4 subgroup has seven members in Arabidopsis, and three of these Raf-like kinases share high homology with each other. Three of the other four members of B4 have not yet been characterised, and the last member, HYDRAULIC CONDUCTIVITY
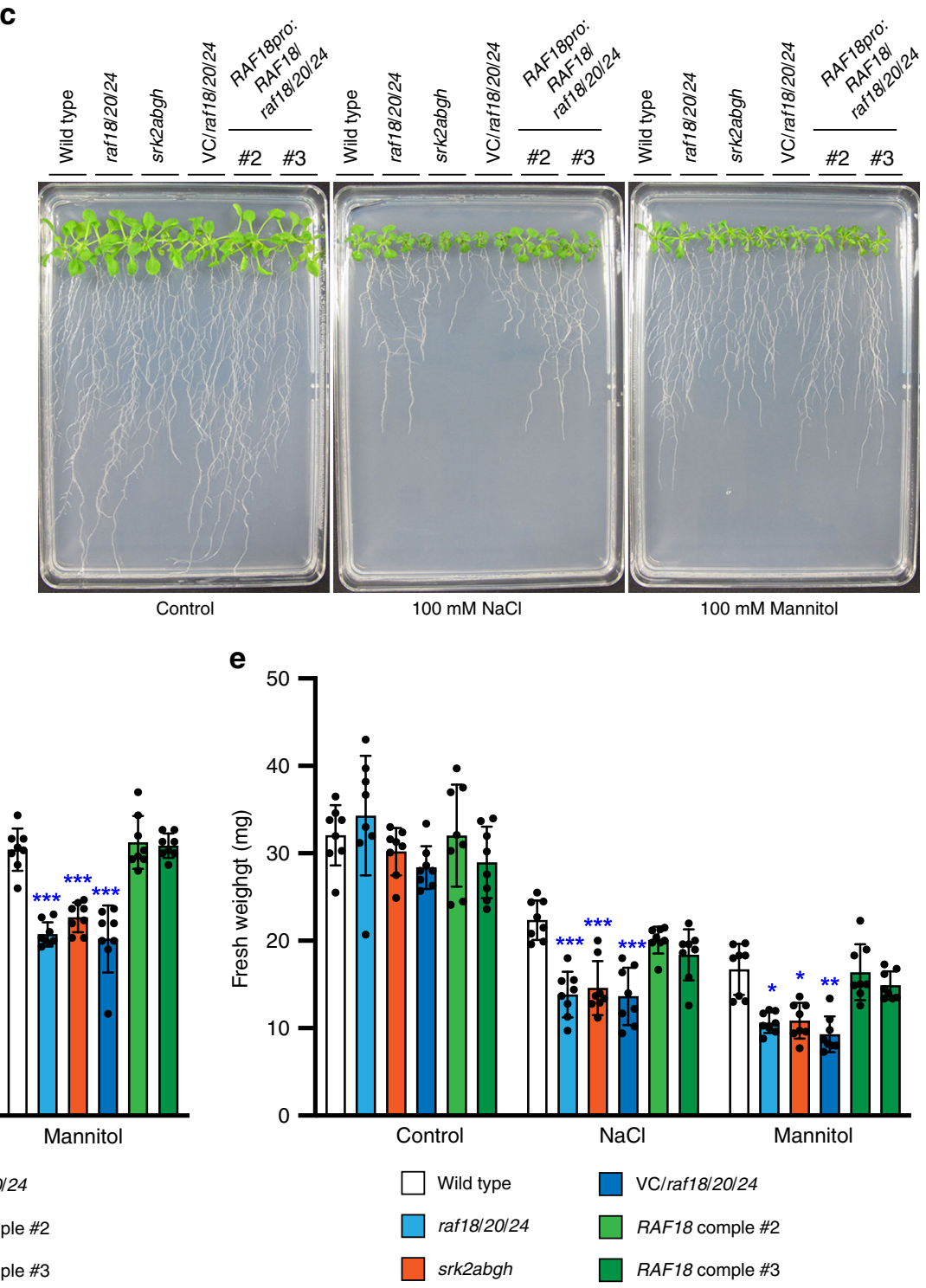

OF ROOT 1 (HCR1), is a negative regulator of root water permeability ${ }^{31}$. Although the B4 subgroup has members found in mosses to seed plants, the three Raf-like kinases are evolutionarily divergent from the members found in lycophytes or mosses (Supplementary Fig. 3). Subclass III SnRK2s are highly conserved from mosses to seed plants, but subclass I SnRK2s have not been 
Fig. 3 The raf18/20/24 mutant displays enhanced growth retardation under osmotic stress conditions. a Growth phenotypes of wild-type, raf18/20/24, srk2abgh, VC/raf18/20/24 (harbouring the vector), and RAF18/raf18/20/24 (harbouring RAF18pro:RAF18) plants grown under water-limited conditions. The plants were grown on germination medium (GM) agar plates for 12 days, in soil for an additional 2 days and subsequently without water for 10 days. b Fresh weight $(\mathrm{mg})$ of the aerial parts of plants grown as described in $\mathbf{a}$. The data are presented as the means \pm s.d.s $(n=12)$. An asterisk shows that the indicated mean was significantly different from the mean value of the wild-type plant under the corresponding condition $\left({ }^{\star} P<0.01,{ }^{\star \star} P<0.001,{ }^{\star \star \star} P<\right.$ 0.0001, two-way ANOVA followed by Tukey's test). c Growth phenotypes of wild-type, raf18/20/24, srk2abgh, vector/raf18/20/24 and RAF18/raf18/20/24 plants grown under high salinity or osmotic stress conditions. The photographs show representative phenotypes of 14-day-old seedlings grown vertically on half-strength Murashige-Skoog agar plates containing $100 \mathrm{mM} \mathrm{NaCl}$ or $100 \mathrm{mM}$ mannitol. d, e Primary root growth (d) and fresh weight (e) of seedlings treated as described in $\mathbf{c}$. The data are presented as the means \pm s.d.s $(n=8)$. An asterisk shows that the indicated mean is significantly different from the mean value of the wild-type plant under the corresponding condition $\left({ }^{\star} P<0.01,{ }^{\star \star} P<0.001,{ }^{\star \star \star} P<0.0001\right.$, two-way ANOVA followed by Tukey's test).

a raf $18 / 20 / 24$
upregulated genes

(400) b

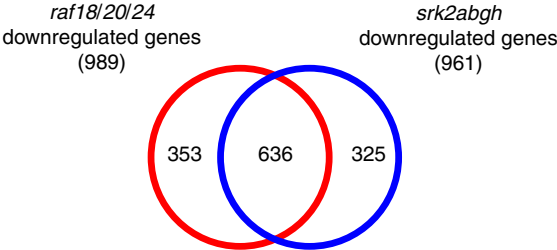

srk2abgh pregulated genes (306)

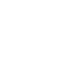

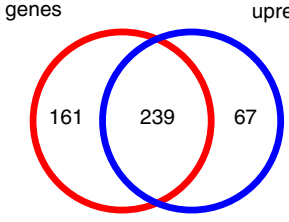

e
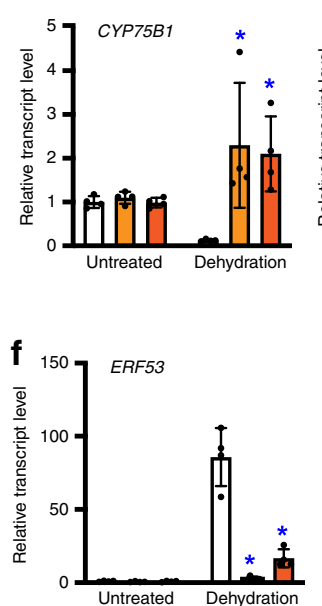

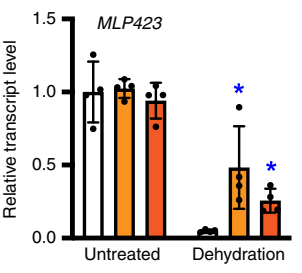

$\square$ Wild type

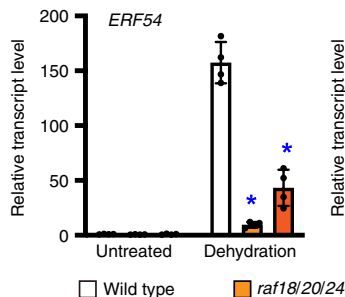

C

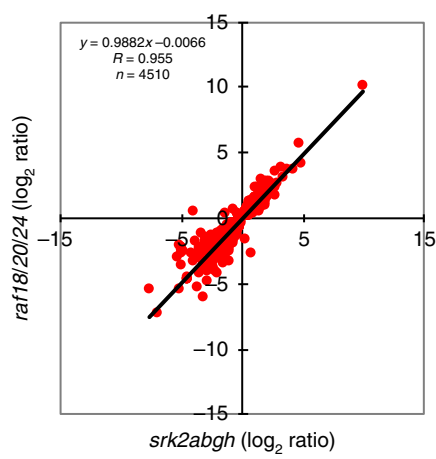

d

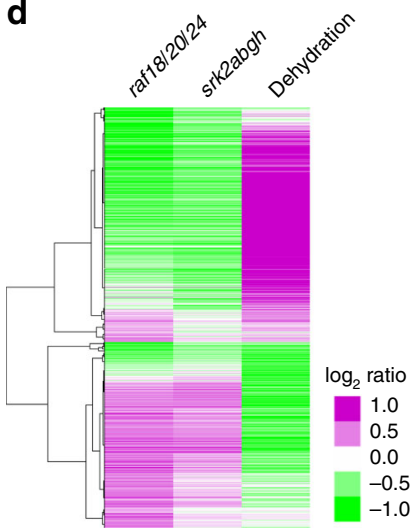

g

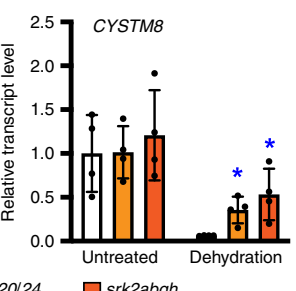

$\square$ srk2abgh

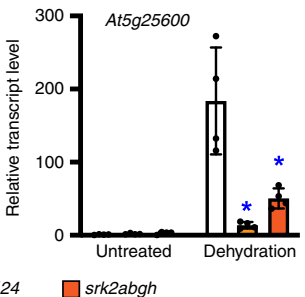

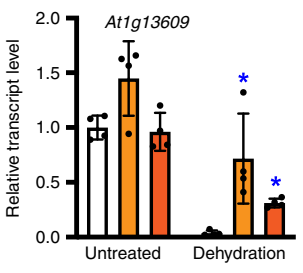

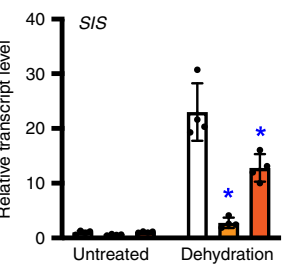

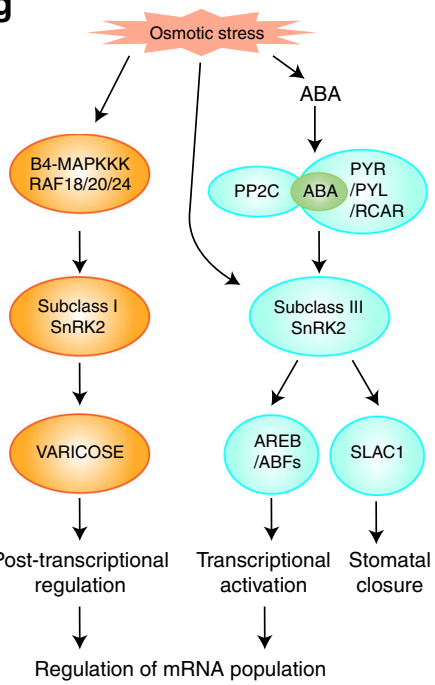

Fig. 4 Transcriptome analysis of RAF18/20/24- and subclass I SnRK2-regulated genes in response to osmotic stress. RAF18/20/24-regulated genes largely overlapped with subclass I SnRK2-regulated genes under dehydration stress conditions. a Venn diagram showing the upregulated genes $(P<0.05$, fold change $>2.0$ ) in the raf18/20/24 and srk2abgh mutants after treatment with dehydration for $5 \mathrm{~h}$. $\mathbf{b}$ Venn diagram showing the downregulated genes $(P<0.05$, fold change $<2.0)$ in the raf18/20/24 and srk2abgh mutants after dehydration treatment for $5 \mathrm{~h}$. $\mathbf{c}$ Analysis of the correlations between the genes downstream of the three RAFs and subclass I SnRK2s. The induction ratios of genes that were significantly changed $(P<0.05)$ in both mutant plants under dehydration are shown in the scatterplots together with the corresponding regression equation and correlation coefficient. $\mathbf{d}$ Hierarchical clustering analysis of RAF18/20/24-regulated genes. Left column: the expression levels of 6804 genes that were significantly up- or downregulated in the raf18/20/ 24 mutant compared with the wild-type expression levels under dehydration conditions. Middle column: the expression levels of the same 6804 genes in the srk2abgh mutant relative to the wild-type expression levels under dehydration conditions. Right column: the wild-type expression levels of the 6804 genes under dehydration stress conditions. e, f Expression levels of upregulated (e) or downregulated genes (f) in the raf18/20/24 and srk2abgh mutants under untreated or dehydration stress conditions. The expression level in the untreated wild-type plants was defined as 1.0. The values are presented as the means \pm s.d.s from four biological replicates. An asterisk shows that the indicated mean was significantly different from the mean value of the wild-type plant under the corresponding condition ( ${ }^{\star} P<0.05$, two-tailed $t$ test with Bonferroni correction). $\mathbf{g}$ Schematic model illustrating that in parallel with transcriptional activation mediated by the "ABA activated subclass III SnRK2-AREB/ABF" signalling pathway, the "RAF18/20/24-subclass I SnRK2VARICOSE" signalling pathway mediates post-transcriptional regulation to control the mRNA population. Three newly identified RAFs are B4 Raf-like MAPKKKs and transduce osmotic stress by phosphorylating subclass I SnRK2s. 
identified in lycophytes and mosses, which is consistent with the finding that members with high homology to the three Raf-like kinase have not been identified in lycophytes or mosses. Subclass I SnRK2s are thought to have been acquired by seed plants to enhance their adaptability to osmotic stress conditions through the control of mRNA accumulation through VCS regulation ${ }^{20}$. The Raf-like kinases appear to be activated by osmotic stress but not ABA, similar to subclass I SnRK2s (Fig. 2). The Raf-like kinases might have evolved with the emergence of the ABAunresponsive subclass I SnRK2-VCS signalling module in seed plants.

In the moss Physcomitrella patens, an ancestral B3-MAPKKK, ABA AND ABIOTIC STRESS-RESPONSIVE RAF-LIKE KINASE (ARK), was previously identified as an upstream factor of ABA-responsive SnRK2s (PpSnRK2s) ${ }^{32}$. ARK activates PpSnRK2s in response to ABA and osmotic stress. All PpSnRK2s encoded in the $P$. patens genome are classified into the subclass III category ${ }^{33}$. A generally accepted mechanism for the activation of subclass III SnRK2 is the following: the binding of ABA to PYR1/PYL/RCAR receptors inhibits group A PP2Cs, which are negative regulators of SnRK2s, and subclass III SnRK2s are thereby activated through autophosphorylation ${ }^{4,5}$. However, it has been reported that the activation of subclass III type PpSnRK2s in response to $\mathrm{ABA}$ requires phosphorylation by the upstream ABA-activated ARK protein kinase in P. patens ${ }^{32}$. Arabidopsis subclass III SnRK2s are known to be activated through both $\mathrm{ABA}$-dependent and ABA-independent pathways ${ }^{16}$. In Arabidopsis, six members belong to the subgroup of B3 Raf-like MAPKKKs (Supplementary Fig. 3), and three of these members complement the activity of ARK in P. patens ${ }^{32}$. Because Arabidopsis B4 Raf-like MAPKKKs specifically activate subclass I SnRK2s in response to osmotic stress, these B3 Raf-like MAPKKKs might activate subclass III SnRK2s in response to $\mathrm{ABA}$ and osmotic stress in an ABA-dependent and/or ABAindependent manner in plants. Indeed, while this paper was under review three B3 Raf-like MAPKKKs were reported to be required for $\mathrm{ABA}$ and osmotic stress-responsive activation of subclass III SnRK2s ${ }^{34}$. Moreover, Arabidopsis Raf10 and Raf11, which belong to the subgroup of B2 MAPKKKs, are reportedly positive regulators of $\mathrm{ABA}$ responses, including the establishment of seed dormancy and glucose/abiotic stress responses, in plants ${ }^{35}$. Although the target proteins of these two kinases have not yet been identified, they might be subclass III SnRK2s because B2 MAPKKKs are more closely related to B3 than B4 MAPKKKs. Further studies using Arabidopsis with various multiple mutations are necessary to clarify the roles of these B2 and B3 Raf-like MAPKKKs in the regulation of the activity of subclass III SnRK2 in plants.

mRNA decapping and subsequent mRNA degradation play important roles in the regulation of the mRNA population to allow adaption to various environmental stress conditions. The activation of mRNA degradation prior to the induction of transcription is considered to result in the activation of stress-induced gene expression and thereby lead to efficient changes in the mRNA population. We previously reported that subclass I SnRK2s play important roles in the regulation of mRNA decay under osmotic stress conditions by phosphorylating the mRNAdecapping activator $\mathrm{VCS}^{20}$. In this study, we revealed that the Raf-like kinases positively regulate the expression of drought stress-responsive genes by activating the subclass I SnRK2-VCS signalling module to promote plant growth under osmotic stress conditions. The growth of the raf18/20/24 and srk2abgh plants and the differentially regulated genes in these mutant plants under osmotic stress conditions were very similar (Figs. 3 and 4), which suggests that the main target proteins of the Raf-like kinases are subclass I SnRK2s and that the activation of the Raf- like kinases is essential for the activation of subclass I SnRK2s under osmotic stress conditions. These results imply that plant growth retardation caused by osmotic stresses, such as drought and salt stress, can potentially be improved by controlling the RAF18/20/24-subclass I SnRK2-VCS signalling pathway.

The next challenge was to identify upstream factors that activate the Raf-like kinases. The factors upstream of a B3 Raf-like MAPKKK, CONSTITUTIVE TRIPLE RESPONSE 1 (CTR1), have been well studied ${ }^{36,37}$. In Arabidopsis, CTR1 negatively regulates ethylene signalling in conjunction with a family of receptors under normal conditions. Upon binding to ethylene, the receptors transmit the signal to the CTR1 protein kinase and thereby inhibit the ability of the kinase to phosphorylate its target protein, ETHYLENE INSENSITIVE 2 (EIN2), which activates downstream ethylene signalling. Ethylene receptors, such as ETHYLENE RESPONSE 1 (ETR1), belong to a family of twocomponent histidine kinases. The set of Arabidopsis histidine kinases consist of 11 members. In plants, five of these members are ethylene receptors, five of the remaining members [CYTOKININ INDEPENDENT (CKI) 1, CKI2, ARABIDOPSIS HISTIDINE KINASE (AHK) 2, AHK3 and AHK4/CYTOKININ RESPONSE 1 (CRE1)] function as cytokinin receptors, and ATHK1 serves as a putative osmosensor ${ }^{38-41}$. Furthermore, complementation experiments with the yeast osmosensor mutant $\sin 1$ and Arabidopsis overexpressor and mutant analyses suggest that the cytokinin receptors AHK2, AHK3, and CRE1/AHK4 have dual functions as osmosensors ${ }^{40}$. Because the sensors upstream of CTR1 are histidine kinases, these candidate osmosensors appear to be factors upstream of the Raf-like kinases. Moreover, upstream factors might be common to subclass III SnRK2s, and the identification of upstream factors of the Raf-like kinases will reveal whether these upstream factors are common or different.

\section{Methods}

Plant materials and generation of transgenic plants. Arabidopsis thaliana (L.) Heynh ecotype Columbia $(\mathrm{Col})$ was used. The seeds were surface-sterilised and sown on plates with germination medium (GM) agar [4.6 g/L Murashige and Skoog Plant Salt Mixture (FUJIFILM Wako Chemicals), $1 \mathrm{~mL} / \mathrm{L}$ GAMBORG'S vitamin solution (SIGMA), $30 \mathrm{~g} / \mathrm{L}$ sucrose, $8.3 \mathrm{~g} / \mathrm{L}$ Bacto Agar (BD Biosciences) and $0.5 \mathrm{~g} / \mathrm{L}$ MES, pH $5.7(\mathrm{KOH})]$. After stratification at $4{ }^{\circ} \mathrm{C}$ for 3 days in the dark, the plates were incubated in a growth chamber under a 16-h light/8-h dark photoperiod $\left(60 \pm 10 \mu \mathrm{E} \mathrm{m}^{-2} \mathrm{~s}^{-1}\right)$ at $22^{\circ} \mathrm{C}$. For plant growth in soil, 12- to 14-day-old seedlings grown on GM agar plates were transferred into a plastic pot (diameter of $8 \mathrm{~cm}$, height of $6.5 \mathrm{~cm}$ ) filled with perlite-containing soil (Dio Chemicals) and grown under a 16-h light $/ 8$-h dark photoperiod $\left(60 \pm 10 \mu \mathrm{E} \mathrm{m}^{-2} \mathrm{~s}^{-1}\right)$ at $22{ }^{\circ} \mathrm{C}$ and $70-85 \%$ relative humidity. The T-DNA insertion lines raf18 (SALK_053373), raf20 (SALK_069912) and raf24 (SALK_107170) were obtained from the Arabidopsis Biological Resource Center (ABRC, Columbus, OH, USA) or the European Arabidopsis Stock Centre (NASC, Nottingham, UK). A series of multiple raf mutants in the Col ecotype were constructed by genetic crosses and screened via genomic PCR using the recommended primers (ABRC). The srk2abgh mutant was constructed by genetic crosses and via genomic $\mathrm{PCR}^{20}$, and the plants were transformed using Agrobacterium tumefaciens strain GV3101 by the floral-dip method $^{42}$. The GV3101 was infiltrated to Nicotiana benthamiana plants for transient expression ${ }^{29,43}$. Five-week-old $N$. benthamiana plants were used for Agrobacterium-mediated transient expression.

Co-immunoprecipitation. Proteins were extracted from approximately 1003 week-old seedlings grown on GM agar plates and treated or not treated with $0.8 \mathrm{M}$ mannitol for $10 \mathrm{~min}$. Crude proteins were extracted in $10 \mathrm{~mL}$ of extraction buffer [20 mM HEPES-KOH (pH 7.6), $100 \mathrm{mM} \mathrm{NaCl}, 0.1 \mathrm{mM}$ EDTA, $5 \mathrm{mM} \mathrm{MgCl}, 20 \%$ (v/v) glycerol and $0.5 \%(\mathrm{v} / \mathrm{v})$ Triton X-100], and subsequently subjected to two-step centrifugation for the removal of cellular debris. The supernatant was incubated with $120 \mu \mathrm{L}$ of $\mu \mathrm{MACS}$ Anti-GFP MicroBeads (130-091-125; Miltenyi Biotec) for $30 \mathrm{~min}$ at $4{ }^{\circ} \mathrm{C}$. The mixtures were applied to M columns (130-042-801; Miltenyi Biotec) placed onto the MiniMACS Separator. The columns were washed with $10 \mathrm{~mL}$ of extraction buffer. Co-immunoprecipitation of mCherry-fused proteins was performed using Anti-RFP mAb-Magnetic Beads (M165-11, MBL) according to the manufacturer's recommended protocol. Transgenic Arabidopsis plants expressing SRK2A-sGFP or SRK2G-sGFP driven by the 35 S promoter were generated by introducing pGH-35Spro:SRK2A or pGH-35Spro:SRK2G into wild-type 
plants, respectively ${ }^{20}$. The SRK2A-GFP and SnRK2G-GFP protein levels were similar in these transgenic plants.

\section{Peptide preparation for tandem mass spectrometry analysis. After electro-} phoresis, immunoprecipitates were fixed and stained with Coomassie brilliant blue. Then, the immunoprecipitates were digested using Trypsin/Lys-C Mix (V5073; Promega) by the in-gel digestion method ${ }^{44}$. The digested peptides were recovered from the gel pieces after adding 50\% (v/v) acetonitrile/5\% (v/v) formic acid and subsequently desalted using GL-Tip SDB and GL-Tip GC columns (GL Sciences, Tokyo, Japan). The extracted peptides were dried in a vacuum evaporator, and subsequently dissolved in $80 \%(\mathrm{v} / \mathrm{v})$ acetonitrile containing $0.1 \%(\mathrm{v} / \mathrm{v})$ formic acid.

Mass spectrometric analysis and database searches. Mass spectrometric analysis was performed using a TripleTOF 5600 instrument (SCIEX) with an Autosampler-2 1D Plus and NanoLC Ultra (Eksigent). Each sample was isolated using a MonoCap C18 High-Resolution 2000 column $(2000 \mathrm{~mm} \times 100-\mu \mathrm{m}$ inside diameter, $2-\mu \mathrm{m}$ pore size; GL Science, Japan). Four microliters of the sample were concentrated through the analytical column at a flow rate of $500 \mathrm{~nL} / \mathrm{min}$ for $30 \mathrm{~min}$. The mobile phase comprised of $2 \%$ acetonitrile and $0.1 \%$ formic acid (A) and $80 \%$ acetonitrile and $0.1 \%$ formic acid (B). The following linear gradient was used in this analysis: $A: B=98: 2$ at 0 min to $A: B=60: 40$ over $300 \min , A: B=10: 90$ over $20 \mathrm{~min}$ and $\mathrm{A}: \mathrm{B}=98: 2$ over $40 \mathrm{~min}$. The MS scan range was a mass/charge ratio $(\mathrm{m} / \mathrm{z})$ of 400 to 1250 , and the top 20 precursor ions were selected for subsequent MS/MS scans in the high-sensitivity mode. The MS/MS data were analysed using ProteinPilot 5.0 software (SCIEX) and subsequently annotated using the $A$. thaliana TAIR10 protein database for peptide identification.

Subcellular localisation and BiFC. A genomic sequence of RAF18 was amplified from the genome extracted from Col-0 and cloned into a pGH-GFP vector ${ }^{11}$ to produce a pGH-RAF18-sGFP vector. A pGKX-DCP1-mCherry plasmid was constructed by inserting the $D C P 1$ coding region into pGKX-mCherry ${ }^{20}$. For BiFC analysis, the genomic sequences of RAF18, RAF20 and RAF24 were inserted into the pSCYCE vector ${ }^{43}$, and the coding sequences of SRK2A and SRK2G were inserted into the pVYNE vector ${ }^{43}$. For fluorescence observations, root or leaf cells were observed under a confocal laser-scanning microscope (LSM5 PASCAL, Carl Zeiss). The primers used for generating the constructs are listed in Supplementary Data 4.

Split-luciferase complementation assays. The genomic sequences of $R A F 18$, RAF20 and RAF24 were inserted into the pCAMBIA-NLuc vector ${ }^{45}$, and a coding sequence of SRK2A was inserted into the pCAMBIA-CLuc vector ${ }^{45}$. Transient expression in $N$. benthamiana leaves was achieved as above. After infiltration of the luciferin reaction solution $\left[0.1 \mathrm{mM}\right.$ luciferin, $10 \mathrm{mM} \mathrm{MgCl} \mathrm{Mn}_{2}$ and $10 \mathrm{mM}$ MES-KOH ( $\mathrm{pH}$ 7.5)], the luciferase activity was detected with an Image Quant LAS-4000 biomolecular imager (GE Healthcare), and the data were visualised using Fiji ${ }^{46}$. The primers used for generating the constructs are listed in Supplementary Data 4.

In-gel kinase assays. Total proteins were extracted from 12-day-old seedlings that were untreated or subjected to dehydration, $0.8 \mathrm{M}$ mannitol or $0.5 \mathrm{M} \mathrm{NaCl}$ treatment for the indicated time periods. To obtain each sample, seedlings from five plants were ground to a powder in liquid nitrogen and homogenised at $4{ }^{\circ} \mathrm{C}$ in $150 \mu \mathrm{L}$ of extraction buffer ( $50 \mathrm{mM}$ HEPES-KOH [pH 7.5], $5 \mathrm{mM}$ EDTA, $5 \mathrm{mM}$ EGTA, $2 \mathrm{mM}$ DTT, $25 \mathrm{mM} \mathrm{NaF}, 1 \mathrm{mM} \mathrm{Na} \mathrm{VO}_{4}, 50 \mathrm{mM} \beta$-glycerophosphate, $20 \%$ $(\mathrm{v} / \mathrm{v})$ glycerol and one tablet of complete ULTRA protease inhibitor cocktail tablet per $25 \mathrm{~mL}$ ). The crude extracts were subsequently centrifuged at $20,000 \mathrm{~g}$ and $4^{\circ} \mathrm{C}$ for $30 \mathrm{~min}$ for the removal of cellular debris. The supernatants were collected and subjected to SDS-PAGE. The gel was washed in washing buffer $[25 \mathrm{mM}$ Tris-HCl $\mathrm{pH} 7.5,5 \mathrm{mM} \mathrm{NaF}, 0.5 \mathrm{~g} / \mathrm{L}$ BSA, $0.1 \mathrm{mM} \mathrm{Na} \mathrm{Vo}_{4}, 0.5 \mathrm{mM}$ DTT, $0.1 \%$ (v/v) Triton $\mathrm{X}-100]$. The gel was further incubated in renaturation buffer $(25 \mathrm{mM}$ Tris- $\mathrm{HCl} \mathrm{pH}$ 7.5, $5 \mathrm{mM} \mathrm{NaF}, 0.1 \mathrm{mM} \mathrm{Na} \mathrm{Vo}_{4}, 1 \mathrm{mM}$ DTT) at $4{ }^{\circ} \mathrm{C}$ overnight. In-gel kinase assays were performed in reaction buffer $(25 \mathrm{mM}$ Tris- $\mathrm{HCl} \mathrm{pH} 7.5,0.1 \mathrm{mM}$ $\mathrm{Na}_{3} \mathrm{Vo}_{4}, 12 \mathrm{mM} \mathrm{MgCl} 2,2 \mathrm{mM}$ DTT, $1 \mathrm{mM}$ EGTA) with $50 \mu \mathrm{Ci}$ [ $\left.\gamma^{-}{ }^{32} \mathrm{P}\right]$-ATP for 90 min at room temperture ${ }^{20,27}$. MBP-tagged VCSm ${ }^{20}$ recombinant protein was used as the substrate. A dephosphorylation assay was performed using $\lambda$-Protein Phosphatase (New England Biolabs) according to the manufacturer's recommended protocol.

Immunoblot analysis. The protein extracts were separated via SDS-PAGE and then subjected to immunoblot analysis ${ }^{27}$. Immunoblotting was performed using a monoclonal anti-GFP (1:2,500 dilution, 11814460001; Roche) or a monoclonal anti-mCherry antibody (1:2000 dilution, [1C51]ab125096; Abcam) as the primary antibody and stabilised peroxidase conjugated goat anti-mouse antibody $(1: 10,000$, 32430 , Thermo Scientific) as the secondary antibody. Signals were developed using the ECL Select Western Blotting Detection Reagent (GE Healthcare) according to the manufacturer's instructions, and detected using the Image Quant LAS-4000 biomolecular imager (GE Healthcare).
In vitro kinase assays. pMAL-c2X-SRK2A, pMAL-c2X-SRK2B, pMAL-c2XSRK2G and pMAL-c2X-SRK2H were constructed by inserting the SRK2A, SRK2B, SRK2G or SRK2H cording region into pMAL-c2X (New England Biolabs) ${ }^{20}$. To generate kinase-negative forms of the SRK2A, SRK2B, SRK2G and SRK2H fragments, site-directed mutagenesis was performed by inverse PCR ${ }^{27,47}$, which resulted in the production of pMAL-c2X-SRK2AKN(K33N), pMAL-c2XSRK2BKN(K33N), pMAL-c2X-SRK2GKN(K33N) and pMAL-c2X-SRK2HKN (K33N), respectively. Fragments of the kinase domains of RAF18 (2499-3402 bp), RAF20 (2802-3681 bp) and RAF24 (2832-3708 bp) were amplified from cDNAs derived from Col-0 plants, and these fragments were cloned into pGEX-4T2 (GE Healthcare Life Sciences) to produce pGEX-4T2-RAF18KD, pGEX-4T2-RAF20KD and pGEX-4T2-RAF24KD, respectively. The resulting plasmids were transformed into Escherichia coli strain BL21-Gold (DE3; Agilent Technologies). The recombinant proteins were expressed in bacteria and affinity purified according to the manufacturer's instructions. For in vitro phosphorylation assays, the purified proteins were incubated in a total volume of $15 \mu \mathrm{L}$ of reaction buffer $(50 \mathrm{mM}$ Tris$\mathrm{HCl} \mathrm{pH} \mathrm{7.5,} 100 \mathrm{mM} \mathrm{NaCl}, 10 \mathrm{mM} \mathrm{MnSO}_{4}, 10 \mathrm{mM} \mathrm{MnCl}_{2}, 0.5 \mathrm{mM} \mathrm{CaCl}_{2}, 2 \mathrm{mM}$ DTT, $\left.10 \mu \mathrm{Ci}\left[\gamma_{-}{ }^{32} \mathrm{P}\right] \mathrm{ATP}\right)$ for $60 \mathrm{~min}$ at $30^{\circ} \mathrm{C}$. The protein samples were then separated by SDS-PAGE, and the gel was subsequently dried and exposed to a Fujifilm imaging plate (BAS-MS; GE Healthcare Life Sciences) for 1d. A radioactive image was visualised by FLA-5000 Phosphor Imager (Fujifilm). The protein level was analysed by Coomassie Brilliant Blue (CBB) staining. The primers used for generating the constructs and site-directed mutagenesis are listed in Supplementary Data 4.

Physiological assays. For the assessment of growth under drought stress conditions, seeds were sown on GM agar plates, stratified at $4{ }^{\circ} \mathrm{C}$ for 3 days in the dark, and subsequently grown under a 16-h light/8-h dark photoperiod $\left(60 \pm 10 \mu \mathrm{E} \mathrm{m}^{-2}\right.$ $\mathrm{s}^{-1}$ ) at $22^{\circ} \mathrm{C}$ for 12 days. The seedlings were transferred from GM agar plates to soil, grown in soil for an additional 2 days, and then treated with or without water for 10 days under $80 \%$ relative humidity. For the assessment of $\mathrm{NaCl}$ or mannitol sensitivity at the seedling stage, seeds were sown on GM agar plates solidified with $1.2 \%(\mathrm{w} / \mathrm{v})$ Bacto agar (BD Biosciences), stratified at $4{ }^{\circ} \mathrm{C}$ for 3 days in the dark, and subsequently grown vertically under a $16-\mathrm{h}$ light/ 8 -h dark photoperiod $(60 \pm$ $10 \mu \mathrm{E} \mathrm{m}^{-2} \mathrm{~s}^{-1}$ ) at $22^{\circ} \mathrm{C}$. Four-day-old seedlings were transferred to fresh agar plates containing half-strength MS medium, $1 \%(\mathrm{w} / \mathrm{v})$ sucrose and $0.5 \mathrm{~g} / \mathrm{L}$ of MES$\mathrm{KOH}$ (pH 5.7) supplemented with $100 \mathrm{mM} \mathrm{NaCl}$ or $100 \mathrm{mM}$ mannitol and solidified with 1.2\% Bacto Agar and subsequently grown vertically for an additional 11 days. Photographs were then obtained, and the relative primary root growth of each plant was determined. The humidity in the Plant Growth Chamber (Nippon Medical \& Chemical Instruments Co., LTD) was controlled according to the manufacturer's recommended protocol.

RNA extraction. The total RNA was prepared from 12-day-old seedlings grown on GM agar plates. Five plants were pooled to obtain a single RNA sample. The extraction of the total RNA from the plants was conducted with RNAiso Plus (Takara Bio). The extracted RNA was subjected to RNA sequencing and RT-qPCR analyses.

RNA sequencing and data analysis. Three independent RNA samples were used as biological replicates in each RNA-sequencing experiment. The total RNA $(1 \mu \mathrm{g})$ was used to prepare each library. mRNA purification was performed using a NEBNext Poly(A) mRNA Magnetic Isolation Module (New England Biolabs). cDNA libraries were constructed using the NEBNext Ultra II RNA Library Kit for Illumina (New England Biolabs) and NEBNext Multiple Oligos for Illumina (New England Biolabs), and the libraries were sequenced by NextSeq 500 (Illumina). The single ends of cDNA libraries were sequenced for $86 \mathrm{nt}$ using the illumine Genome Analyzer $\mathrm{IIx}^{48}$. The reads were mapped to the Arabidopsis reference (TAIR10). A heatmap was generated using Gene Cluster $3.0^{49}$ and Java TreeView ${ }^{50}$ to visualise the clustering results.

Quantitative RT-PCR. Four independent RNA samples were used as biological replicates in each quantitative RT-PCR experiment. First-strand cDNA was synthesised from $1 \mu \mathrm{g}$ of the total RNA using a High-Capacity cDNA Reverse Transcription Kit (Thermo Fisher Scientific, Waltham, MA, USA) with random hexamer primers according to the manufacturer's instructions. Quantitative RTPCR was performed using QuantStudio 3 Real-time PCR (Applied Biosystems), and Power SYBR Green PCR Master Mix (Applied Biosystems) was used for amplification. Arabidopsis $18 \mathrm{~S}$ rRNA was used as an internal control in the quantitative RT-PCR assay. The primers used for real-time PCR are listed in Supplementary Data 4.

Accession numbers. Sequences of genes described in this article can be found in The Arabidopsis Information Resource (http://www.arabidopsis.org/) under the following accession numbers: SRK2A/SnRK2.4 (At1g10940), SRK2G/SnRK2.1 (At5g08590), SRK2B/SnRK2.10 (At1g60940), SRK2H/SnRK2.5 (At5g63650), SRK2D/SnRK2.2 (At3g50500), VCS/VARICOSE (At3g13300), DCP1 (At1g08370), DCP2 (At5g13570), RAF18 (At1g16270), RAF20 (At1g79570), RAF24 
(At2g35050), CYP75B1 (At5g07990), MLP423 (At1g24020), CYSTM8 (At3g22235), ERF53 (At2g20880), ERF54 (At4g28140) and SIS (At5g02020).

Reporting summary. Further information on research design is available in the Nature Research Reporting Summary linked to this article.

\section{Data availability}

The LC-MS/MS data of SnRK2-interacting proteins have been deposited to the ProteomeXchange Consortium via the PRIDE ${ }^{51}$ partner repository with the dataset identifier PXD017371. The RNA-sequencing data were deposited into the DNA Data Bank of Japan under accession number DRA008643. The source data underlying Figs. 1b 2a-h, 3b, d, e, and 4e, f and Supplementary Figs 4b, 5, 6b, 7b, d-e, 8a-b, 9a-b, 10b, 11b, $12 \mathrm{~b}, 13 \mathrm{~b}, 13 \mathrm{~b}-\mathrm{c}$ and $14 \mathrm{~b}$ are provided as a Source Data file.

Received: 29 July 2019; Accepted: 18 February 2020;

Published online: 13 March 2020

\section{References}

1. Mustilli, A. C., Merlot, S., Vavasseur, A., Fenzi, F. \& Giraudat, J. Arabidopsis OST1 protein kinase mediates the regulation of stomatal aperture by abscisic acid and acts upstream of reactive oxygen species production. Plant Cell 12, 3089-3099 (2002).

2. Yoshida, R. et al. ABA-activated SnRK2 protein kinase is required for dehydration stress signaling in Arabidopsis. Plant Cell Physiol. 43, 1473-1483 (2002).

3. Boudsocq, M., Barbier-Brygoo, H. \& Laurière, C. Identification of nine sucrose nonfermenting 1-related protein kinases 2 activated by hyperosmotic and saline stresses in Arabidopsis thaliana. J. Biol. Chem. 279, 41758-41766 (2004).

4. Park, S. Y. et al. Abscisic acid inhibits type $2 \mathrm{C}$ protein phosphatases via the PYR/PYL family of START proteins. Science 324, 1068-1071 (2009).

5. Ma, Y. et al. Regulators of PP2C phosphatase activity function as abscisic acid sensors. Science 324, 1064-1068 (2009).

6. Nishimura, N. et al. Structural mechanism of abscisic acid binding and signaling by dimeric PYR1. Science 326, 1373-1379 (2009).

7. Santiago, J. et al. The abscisic acid receptor PYR1 in complex with abscisic acid. Nature 462, 665-668 (2009).

8. Miyazono, K. et al. Structural basis of abscisic acid signalling. Nature 462, 609-614 (2009).

9. Melcher, K. et al. A gate-latch-lock mechanism for hormone signalling bny abscisic acid receptors. Nature 462, 602-608 (2009).

10. Yin, P. et al. Structural insights into the mechanism of abscisic acid signaling by PYL proteins. Nat. Struct. Mol. Biol. 16, 1230-1236 (2009).

11. Furihata, T. et al. Abscisic acid-dependent multisite phosphorylation regulates the activity of a transcription activator AREB1. Proc. Natl Acad. Sci. USA 103 1988-1993 (2006).

12. Fujii, H. \& Zhu, J. K. Arabidopsis mutant deficient in 3 abscisic acid-activated protein kinases reveals critical roles in growth, reproduction, and stress. Proc. Natl Acad. Sci. USA 106, 8380-8385 (2009).

13. Fujita, Y. et al. Three SnRK2 protein kinases are the main positive regulators of abscisic acid signaling in response to water stress in Arabidopsis. Plant Cell Physiol. 50, 2123-2132 (2009).

14. Fujii, $\mathrm{H}$. et al. In vitro reconstitution of an abscisic acid signalling pathway. Nature 462, 660-664 (2009).

15. Umezawa, T. et al. Type $2 \mathrm{C}$ protein phosphatases directly regulate abscisic acid-activated protein kinases in Arabidopsis. Proc. Natl Acad. Sci. USA 106, 17588-17593 (2009)

16. Yoshida, R. et al. The regulatory domain of RK2E/OST1/SnRK2.6 interacts with ABI1 and integrates abscisic acid (ABA) and osmotic stress signals controlling stomatal closure in Arabidopsis. J. Biol. Chem. 281, 5310-5318 (2006).

17. Fujii, H., Verslues, P. E. \& Zhu, J. K. Arabidopsis decuple mutant reveals the importance of SnRK2 kinases in osmotic stress responses in vivo. Proc. Natl Acad. Sci. USA 108, 1717-1722 (2011).

18. McLoughlin, F. et al. The Snf1-related protein kinases SnRK2.4 and SnRK2.10 are involved in maintenance of root system architecture during salt stress. Plant J. 72, 436-449 (2012).

19. Maszkowska, J. et al. Phosphoproteomic analysis reveals that dehydrins ERD10 and ERD14 are phosphorylated by SNF1-related protein kinase 2.10 in response to osmotic stress. Plant Cell Environ. 42, 931-946 (2019).

20. Soma, F. et al. ABA-unresponsive SnRK2 protein kinases regulate mRNA decay under osmotic stress in plants. Nat. Plants 3, 16204 (2017)

21. Deyholos, M. K. et al. VARICOSE, a WD-domain protein, is required for leaf blade development. Development 130, 6577-6588 (2003).
22. $\mathrm{Xu}$, J. et al. Arabidopsis DCP2, DCP1, and VARICOSE form a decapping complex required for postembryonic development. Plant Cell 18, 3386-3398 (2006).

23. Umezawa, T. et al. Molecular basis of the core regulatory network in ABA responses: sensing, signaling and transport. Plant Cell Physiol. 51, 1821-1839 (2010).

24. Boudsocq, M. et al. Different phosphorylation mechanisms are involved in the activation of sucrose non-fermenting 1 related protein kinases 2 by osmotic stresses and abscisic acid. Plant Mol. Biol. 63, 491-503 (2007).

25. Stecker, K. E., Minkoff, B. B. \& Sussman, M. R. Phosphoproteomic analyses reveal early signaling events in the osmotic stress response. Plant Physiol. 165, 1171-1187 (2014)

26. Ichimura, K. et al. Mitogen-activated protein kinase cascades in plants: a new nomenclature. Trends Plant Sci. 7, 301-308 (2002).

27. Mogami, J. et al. Two distinct families of protein kinases are required for plant growth under high external $\mathrm{Mg} 2+$ concentrations in Arabidopsis. Plant Physiol. 167, 1039-1057 (2015).

28. Zheng, C. F. \& Guan, K. L. Activation of MEK family kinases requires phosphorylation of two conserved Wer/Thr residues. EMBO J. 13, 1123-1131 (1994).

29. Vlad, F. et al. Phospho-site mapping, genetic and in planta activation studies reveal key aspects of the different phosphorylation mechanisms involved in activation of SnRK2s. Plant J. 63, 778-790 (2010).

30. Colcombet, J. \& Hirt, H. Arabidopsis MAPKs: a complex signalling network involved in multiple biological processes. Biochem. J. 413, 217-226 (2008).

31. Shahzad, Z. et al. A potassium-dependent oxygen sensing pathway regulates plant root hydraulics. Cell 167, 87-98 (2016).

32. Saruhashi, M. et al. Plant Raf-like kinase integrates abscisic acid and hyperosmotic stress signaling upstream of SNF1-related protein kinase2. Proc. Natl Acad. Sci. USA 112, E6388-kinase6396 (2015).

33. Shinozawa, A. et al. SnRK2 protein kinases represent an ancient system in plants for adaptation to a terrestrial environment. Commun. Biol. 2, 30 (2019)

34. Takahashi, Y. et al. MAP3Kinase-dependent SnRK2-kinase actibvation is required for abscisic acie signal transduction and rapid osmotic stress response. Nat. Commun. 11, 12 (2020).

35. Lee, S. J. et al. Arabidopsis putative MAP kinase kinase kinases Raf10 and Raf11 are positive regulators of seed dormancy and ABA response. Plant Cell Physiol. 56, 84-97 (2014)

36. Guo, H. \& Ecker, J. R. The ethylene signaling pathway: new insights. Curr. Opin. Plant Biol. 27, 40-49 (2004).

37. Yoo, S. D., Cho, Y. H., Tena, G., Xiong, Y. \& Sheen, J. Dual control of nuclear EIN3 by bifurcate MAPK cascades in $\mathrm{C}_{2} \mathrm{H}_{4}$ signalling. Nature 451, 789-795 (2008).

38. Inoue, $\mathrm{T}$. et al. Identification of CRE1 as a cytokinin receptor from Arabidopsis. Nature 409, 1060-1063 (2001).

39. Urao, T., Yamaguchi-Shinozaki, K. \& Shinozaki, K. Plant histidine kinases: an emerging picture of two-component signal transduction in hormone and environmental responses. Sci. Stke. 109, re18 (2001).

40. Tran, L.-S. P. et al. Functional analysis of AHK1/ATHK1 and cytokinin receptor histidine kinases in response to abscisic acid, drought, and salt stress in Arabidopsis. Proc. Natl Acad. Sci. USA 104, 20623-20628 (2007).

41. Wohlbach, D. J., Quirino, B. F. \& Sussman, M. R. Analysis of the Arabidopsis histidine kinase ATHK1 reveals a connection between vegetative osmotic stress sensing and seed maturation. Plant Cell 20, 1101-1117 (2008).

42. Clough, S. J. Floral dip: agrobacterium-mediated germ line transformation. Methods Mol. Biol. 286, 91-102 (2005).

43. Waadt, R. et al. Multicolor bimolecular fluorescence complementation reveals simultaneous formation of alternative CBL/CIPK complexes in planta. Plant J. 56, 505-516 (2008).

44. Shevchenko, A., Tomas, H., Havlis, J., Olsen, J. V. \& Mann, M. In-gel digestion for mass spectrometric characterization of proteins and proteomes. Nat. Protoc. 1, 2856-2860 (2006).

45. Chen, H. et al. Firefly luciferase complementation imaging assay for proteinprotein interactions in plants. Plant Physiol. 146, 368-376 (2008).

46. Schindelin, J. et al. Fiji: an open-source platform for biological-image analysis. Nat. Methods 9, 676-682 (2012).

47. Imai, Y., Matsushima, Y., Sugimura, T. \& Terada, M. A simple and rapid method for generating a deletion by PCR. Nucleic Acids Res. 19, 2785 (1991)

48. Notaguchi, M., Higashiyama, T. \& Suzuki, T. Identification of mRNAs that move over long distances using an RNA-Seq analysis of Arabidopsis/ Nicotiana benthamiana heterografts. Plant Cell Physiol. 56, 311-321 (2015).

49. de Hoon, M. J., Imoto, S., Nolan, J. \& Miyano, S. Open source clustering software. Bioinformatics 20, 1453-1454 (2004).

50. Saldanha, A. J. Java Treeview-extensible visualization of microarray data. Bioinformatics 20, 3246-3248 (2004).

51. Perez-Riverol, Y. et al. The PRIDE database and related tools and resources in 2019: improving support for quantification data. Nucleic Acids Res. 47(D1), D442-D450 (2019). 


\section{Acknowledgements}

The authors would like to thank Yuriko Tanaka, Saho Mizukado, Tomomi Shinagawa and Ayumi Furuta for the excellent technical assistance provided and Etsuko Toma for the skilled editorial assistance provided. This work was financially supported by Grantsin-Aid for JSPS Fellows (No. 18J13854 to F. Soma), Scientific Research on Innovative Areas (No. 15 H05960 to K.Y.-S.), and Scientific Research (A) (No. 18 H03996 to K.Y.-S.) from the Ministry of Education, Culture, Sports, Science, and Technology of Japan and for Scientific Research from the Mitsubishi Foundation.

\section{Author contributions}

F.S. and K.Y.-S. designed the research. F.S. performed most of the experiments. F.T performed the mass spectrometric analysis and database searches. T.S. performed the RNA sequencing and data analyses. F.S., K.S. and K.Y.-S. wrote the article. All authors discussed the results and commented on the paper.

\section{Competing interests}

The authors declare no competing interests.

\section{Additional information}

Supplementary information is available for this paper at https://doi.org/10.1038/s41467020-15239-3

Correspondence and requests for materials should be addressed to K.Y.-S.
Peer review information Nature Communications thanks Claudius Marondedze and the other, anonymous, reviewer(s) for their contribution to the peer review of this work. Peer reviewer reports are available.

Reprints and permission information is available at http://www.nature.com/reprints

Publisher's note Springer Nature remains neutral with regard to jurisdictional claims in published maps and institutional affiliations.

(i) Open Access This article is licensed under a Creative Commons Attribution 4.0 International License, which permits use, sharing, adaptation, distribution and reproduction in any medium or format, as long as you give appropriate credit to the original author(s) and the source, provide a link to the Creative Commons license, and indicate if changes were made. The images or other third party material in this article are included in the article's Creative Commons license, unless indicated otherwise in a credit line to the material. If material is not included in the article's Creative Commons license and your intended use is not permitted by statutory regulation or exceeds the permitted use, you will need to obtain permission directly from the copyright holder. To view a copy of this license, visit http://creativecommons.org/ licenses/by/4.0/.

(c) The Author(s) 2020 